\title{
Is therapy still a matter of chemistry?
}

\begin{abstract}
Liberati DE*
Dipartimento di Elettronica, Research Director National Research Council of Italy, Milano, Italy

Traditionally, therapy mainly reminds of drugs, and drug mainly refer to chemistry: this is of course still widely true! But: Health and well-being is a state of dynamical equilibrium of a complex system, our organism: on an abstract level, it is thus a question of correct information. In fact, the basic chemistry on which we are build -the DNA - is written and read in chemistry, but represents the information program allowing all of us to become ourselves by interacting with the surrounding. A disease is a malfunctioning in such a program, a therapy is a control effort to reconduct the damaged system to a dynamical state less compromised than the one caused by the disease.
\end{abstract}

One of the therapies is usually still the proper drug, possibly tailored according to the very "shape" of the subject in the so called personalized medicine. In a sense the application field of the drugs is even growing: Psichiatry, formerly mostly behavioural, has then evoluted to mostly chemical. In the opposite sense in oncology chemotherapy has been

Copyright: $(02017$ Liberati DE. This is an open-access article distributed under the terms of the Creative Commons Attribution License, which permits unrestricted use, distribution, and reproduction in any medium, provided the original author and source are credited. paired by radiotherapy and psychotherapy. In general, the (hopefully personalized) drug is just one piece of a more complex approach, including physical and other tools.

Moreover, even drug discovery is also partially shifting toward information technology: data mining approaches are current in order to predict hydrophilic behaviour of a candidate new drug, thus speeding and making cheaper the initial steps of the discovery process. Personalizing is again an information process, trying to match drug properties to genetic odds of each patient.

Chemistry is thus still the - or at least a - princess of therapy, not only but still mainly through drugs, not only in the sense of providing active principles avoiding unwilled bad effects, but also conveys information in order to restore the biological system, and exploits information theory tools in order to design drugs, within the frame of the so-called systems biology (Sacco, Farina et al., Biotechnology Advances 2012). 